\title{
Taxonomic Assessment of the Ctenophorus decresii Complex (Reptilia: Agamidae) Reveals a New Species of Dragon Lizard from Western New South Wales
}

\author{
Claire A. McLean ${ }^{1,2 *}$, Adnan Moussalli ${ }^{2}$, Steve Sass $^{3,4}$, and Devi Stuart-Fox ${ }^{1}$ \\ ${ }^{1}$ Department of Zoology, The University of Melbourne, Parkville VIC 3010, Australia \\ ${ }^{2}$ Sciences Department, Museum Victoria, Carlton Gardens VIC 3053, Australia \\ ${ }^{3}$ EnviroKey, PO Box 7231, Tathra NSW 2550, Australia \\ ${ }^{4}$ Institute for Land, Water and Society, Charles Sturt University, Thurgoona NSW 2640, Australia \\ mcleanca@unimelb.edu.au
}

\begin{abstract}
We describe a new species of agamid lizard, Ctenophorus mirrityana sp.nov. currently known from two disjunct populations in western New South Wales. The species is a member of the $C$. decresii species complex, and was formerly recognized as an outlying population of $C$. decresii due to similarities in dorsal colour pattern and adjacent distributions. Previous work documented deep molecular divergence, across multiple loci, with no genetic admixture between the new species and proximal $C$. decresii populations. We find that the new species differs in morphology from all other members of the species complex and is characterized by distinct male throat and lateral coloration, a small head size relative to snout-vent length, a large number of labial scales, and a lack of tubercular scales. We also identify two geographically structured lineages (northern and southern) within C. decresii as requiring further taxonomic investigation, based on notable genetic and morphological (including colour) divergence. We find that divergence in coloration is associated with genetic and body form differentiation within the $C$. decresii species complex.
\end{abstract}

\begin{abstract}
Mclean, Claire A., Adnan Moussalli, Steve Sass, and Devi Stuart-Fox. 2013. Taxonomic assessment of the Ctenophorus decresii complex (Reptilia: Agamidae) reveals a new species of Dragon Lizard from western New South Wales. Records of the Australian Museum 65(3): 51-63.
\end{abstract}

KeYwords: Agamidae; Barrier Range; colour variation; Ctenophorus mirrityana; reptilian morphology

Ctenophorus is the most diverse Australian genus of agamid lizards, comprising 28 small to moderate sized, dry to arid adapted species (Houston \& Hutchinson, 1998; Wilson \& Swan, 2010). Within South Australia (SA), the Ctenophorus decresii complex consists of four closely related, rockinhabiting species: C. decresii (Duméril \& Bibron 1837),
C. fionni (Procter 1923), C. tjantjalka Johnston 1992, and C. vadnappa (Houston 1974), with C. rufescens (Stirling \& Zietz 1893) as a sister clade to the group (Melville et al., 2001; Chen et al., 2012). All species are sexually dimorphic with cryptically coloured females and larger, brightly coloured males which perform conspicuous courtship and

\footnotetext{
* author for correspondence
} 